\title{
Biomarkers in clinical drug development: realizing the promise
}

\begin{abstract}
"Whether through academic research, precompetitive consortia or internal company efforts, there must be a concerted effort to identify, qualify, validate and standardize assays for such biomarkers in most human diseases."
\end{abstract}

Over the years, a variety of new technologies and approaches to drug discovery and development have been touted as having the potential to 'revolutionize' the pharmaceutical industry. High-throughput screening, combinatorial chemistry, various omics technologies, the human genome sequence, RNA silencing techniques, pharmacokinetic (PK)/pharmacodynamic (PD) modeling all come to mind in this regard. All have had an evolutionary impact, but arguably none has been truly revolutionary. The latest bandwagon to leave this station is 'translational medicine' (TM) and biomarkers - the former being loosely defined as the discipline responsible for developing the latter. What impact will this latest trend have on the declining productivity of the industry?

By now, everyone is familiar with the standard definition of a biomarker as "a characteristic that is measured and evaluated as an indicator of normal biological processes, pathogenic processes or pharmacological responses to a therapeutic intervention." A cynical observer might note that biomarkers have been around for a long time, but were not quite as 'sexy' when merely called 'pharmacodynamics'. However, classical PD end points have largely been concerned with direct biochemical/physiologic effects of drugs on their intended targets (e.g., enzyme inhibition, receptor antagonism) - independent of their relationship to potential clinical benefit. While these $\mathrm{PD}$ end points surely meet the definition of a biomarker, the TM approach involves developing (often concomitantly with a new drug) and using two relatively new classes of biomarker.

The first might be called an 'outcome' biomarker - a marker that can be used as a surrogate for clinical benefit, if not for registrational purposes, then at least for internal program decisions. The use of such markers is driven by the vast expense and time required to shepherd a drug through the clinical development process, as well as the extremely high attrition rates. This has led to a need for objective end points that allow a clinical trial sponsor to quickly evaluate whether or not an experimental drug is at least 'reasonably likely' to produce the requisite clinical benefit, so as to more rapidly terminate the losers and direct the saved resources towards more promising candidates in the pipeline. A good example of such outcome biomarkers might be the variety of imaging end points employed in oncology drug studies (e.g., PET scans, or even traditional RECIST-based radiographic measures of tumor size) that are intended as earlier substitutes for the lengthy survival studies usually required for marketing approval. It is now becoming commonplace in many therapeutic areas to see small Phase IIa studies of new drugs, performed in selected patients at or near the maximum tolerated dose, using biomarker-based primary end points in order to drive the go/no-go decision to larger Phase IIb dose-ranging studies, or even pivotal Phase III studies. Of course, the level of confidence in any such biomarker-driven decision is dependent on the level of qualification and validation of the biomarker itself.

\section{"A cynical observer might note that biomarkers have been around for a long time, but were not quite as 'sexy' when merely called 'pharmacodynamics'."}

The distinction between a traditional PD end point and an outcome biomarker is not hard and fast, and increasingly, early project progression decisions are made on PD considerations. It has long been standard practice in the industry to make decisions based on PKs. That is, a 'projected efficacious exposure' is defined based on animal models of the intended disease, and extrapolated to humans using allometric scaling algorithms. Drugs can be terminated in Phase II

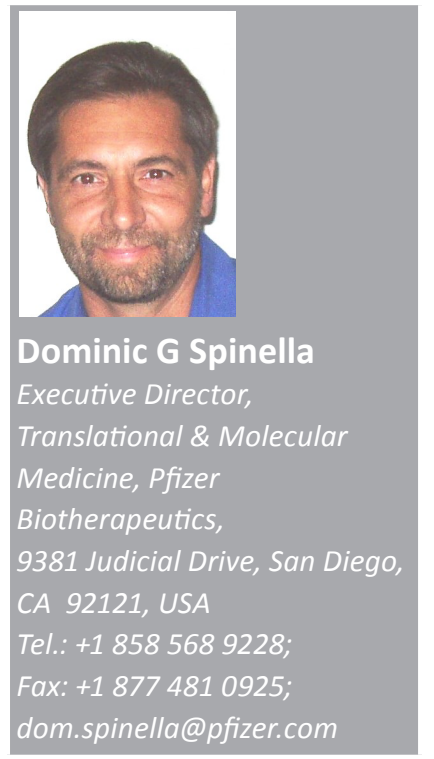

future medictio $^{\text {pisg }}$ 
dose-escalation studies if unacceptable toxicities are encountered before reaching the projected efficacious exposure. It is also possible, however, to determine the extent (and duration) of PD modulation of a drug target that is associated with efficacy in the models, and extrapolate this to clinical studies - terminating drugs in which unacceptable toxicity is encountered at a dose below that necessary for sufficient modulation of the PD end point. Indeed, it might be argued that drug-target modulation is a more appropriate metric than mere drug exposure, and the relationship between PDs and outcome may be better conserved between species than PKs and outcome. The best approach is likely to incorporate both PK and PD data, which is why PK/ PD modeling has assumed importance in early drug development, both in helping to make go/ no-go decisions as well as to define dose range. In any case, what is required is a biomarker that truly reflects human target modulation, preferably at the intended site of action, that can be reasonably assessed in clinical studies.

\section{"The enthusiasm for developing both classes of biomarkers is driven not only by the need to make quicker and cheaper decisions, but also by the proliferation of new technologies...'}

The second new class of biomarker might be called a 'patient-selection' marker. Such a marker, or sometimes a composite or signature of multiple markers, is intended to identify those patients who are more or less likely to respond (or experience adverse effects) to a given drug, facilitating a personalized approach to drug delivery. A growing number of such markers have been identified and have even been incorporated into drug labels. Examples include Her2/Neu levels for the use of trastuzumab in breast cancer, or CYP2C9 and VKORC1 variants for the use of warfarin. While such markers are often cited as prototypical examples of personalized medicine, one might argue that the current crop of patientselection biomarkers are relatively obvious from a pharmacologic perspective: a patient who does not express the presumed drug target, or has a variant that alters its interaction with that drug, is likely to respond to the drug differently, if at all. A patient with low expression, or a poorly active variant, of a drug-metabolizing enzyme is more likely to be overexposed to a drug that is eliminated by that enzyme. These are hardly surprising propositions. However, such biomarkers, even where they exist, typically account for only a fraction of the variability in drug responsiveness or toxicity. It is much more difficult to elucidate molecular factors that are not so directly related to variations in target expression or structure per se, or to polymorphisms in metabolizing enzymes involved in clearance. Nevertheless, apart from the obvious benefit to patients of avoiding exposure to unhelpful or harmful drugs, the utility of such markers to the industry is increased probability of registrational success in studies of smaller subpopulations whose average clinical benefit is higher.

The enthusiasm for developing both classes of biomarkers is driven not only by the need to make quicker and cheaper decisions, but also by the proliferation of new technologies that enable relatively rapid discovery of molecular markers that are at least correlated with drug-response phenotypes. These hypothesis-independent approaches to biomarker discovery are in widespread use. Many clinical studies prospectively collect tissue or blood samples from as many subjects as possible for gene expression, mutation/polymorphism analysis, proteomic analysis or even whole-genome scans in order to establish correlations with eventual outcome.

While these large-scale molecular technologies are extraordinarily powerful, there remain substantial difficulties in applying them to clinical studies. Among these is the problem of data over-fitting. It is intuitively obvious that if one collects tens or hundreds of thousands of unrelated bits of information about two relatively small groups of individuals (e.g., good vs poor responders), one can usually find post facto a subset that appears to discriminate between the groups, simply by random chance. That these bits of information are gene transcript levels or SNPs instead of things such as favorite colors or postal codes, does not necessarily make the apparent correlations any less spurious. This problem is only partially ameliorated by the training-set/test-set designs that are now in routine use, and importantly, is greatly exacerbated by the small samples sizes found in early clinical studies.

Putative biomarkers or predictive signatures resulting from such analyses in small numbers of patients must be considered as no more than hypotheses that must be tested in appropriately designed follow-up studies. This, in turn, often results in the process of biomarker qualification and validation lagging behind the drugdevelopment program it is intended to support. Hence, by the time a biomarker signature is shown to be truly predictive of patient outcome 
(qualified), and an assay with appropriate operating characteristics is developed (validated), often the parent drug has either already failed a pivotal trial in all-comers, or has received marketing approval based on its overall activity. In the latter case, postmarket identification of a more-or-less responsive patient subset may result in a label revision, but is unlikely to change reimbursement. Moreover, it is rare that a failed pivotal study is repeated in a molecularly defined patient subset, or otherwise rescued by biomarkers, as there is often insufficient time to drug loss of exclusivity to render such a do-over commercially viable.

\section{"Even biomarkers with negative-predictive value ... can be enormously valuable tools in the development of new medicines..."}

What then are possible solutions to these problems that might allow TM and biomarkers to realize their full potential to the pharmaceutical industry? First, I would argue that that process of discovering, qualifying and validating outcome biomarkers for diseases should be a recognized, valued and funded discipline - independently of new drug studies. Even biomarkers with negativepredictive value (i.e., patients who fail to show a biomarker effect rarely derive clinical benefit, while only a fraction of those who do show the effect will ultimately derive benefit) can be enormously valuable tools in the development of new medicines, despite lacking sufficient positivepredictive value to achieve true surrogacy status. Consider, for example, the impact on oncology drug development of the RECIST guidelines for assessment of drug effects on tumor size even though tumor size reduction is an imperfect surrogate for survival. Whether through academic research, precompetitive consortia or internal company efforts, there must be a concerted effort to identify, qualify, validate and standardize assays for such biomarkers in most human diseases. It is encouraging to observe that all these things are beginning to happen.
Second, I would argue that discovery and validation of patient-selection markers and molecular signatures to predict drug response or toxicity are only rarely possible until sufficient numbers of patients have been exposed and analyzed. Often this will involve postmarket studies and retrospective analyses. Even the best drugs work in only some of the patients who take them, and virtually all drugs have unwanted side effects in at least a few patients. There needs to be incentive for companies to continue to elucidate molecular predictors of efficacy and toxicity for their drugs post-market - whether this be increased reimbursement or increased time of exclusivity. Similar incentives could also be applied to the re-testing of failed Phase III drugs so that potentially promising new medicines are not left on the shelf solely for commercial reasons relating to patent life.

So, will the widespread adoption of biomarkers and TM approaches revolutionize the Pharma industry and dramatically enhance productivity? Perhaps that is a bit much to expect. A successful drug ultimately requires many stars to align: identification of the right target; production of a chemical or biological entity with the right pharmacological and toxicological properties; development of the right formulation and manufacturing process; choice of the right dose and schedule; and execution of the right clinical development program in the right patients - and perhaps a good deal of sheer luck as well! Still, in this era of increasing pressure on the industry from all sides, biomarkers can be valuable tools for making informed decisions along a long and arduous road, and most companies are enthusiastically jumping on the bandwagon.

\section{Financial \& competing interests disclosure}

The author is an employee of Pfizer, Inc. The author has no other relevant affiliations or financial involvement with any organization or entity with a financial interest in or financial conflict with the subject matter or materials discussed in the manuscript apart from those disclosed.

No writing assistance was utilized in the production of this manuscript. 